\title{
Surrogate Modeling for Uncertainty Assessment with Application to Aviation Environmental System Models
}

\author{
D. Allaire* and K. Willcox \\ Massachusetts Institute of Technology, Cambridge, Massachusetts 02139
}

DOI: $10.2514 / 1 . J 050247$

\begin{abstract}
Numerical simulation models to support decision-making and policy-making processes are often complex, involving many disciplines, many inputs, and long computation times. Inputs to such models are inherently uncertain, leading to uncertainty in model outputs. Characterizing, propagating, and analyzing this uncertainty is critical both to model development and to the effective application of model results in a decision-making setting; however, the many thousands of model evaluations required to sample the uncertainty space (e.g., via Monte Carlo sampling) present an intractable computational burden. This paper presents a novel surrogate modeling methodology designed specifically for propagating uncertainty from model inputs to model outputs and for performing a global sensitivity analysis, which characterizes the contributions of uncertainties in model inputs to output variance, while maintaining the quantitative rigor of the analysis by providing confidence intervals on surrogate predictions. The approach is developed for a general class of models and is demonstrated on an aircraft emissions prediction model that is being developed and applied to support aviation environmental policy-making. The results demonstrate how the confidence intervals on surrogate predictions can be used to balance the tradeoff between computation time and uncertainty in the estimation of the statistical outputs of interest.
\end{abstract}

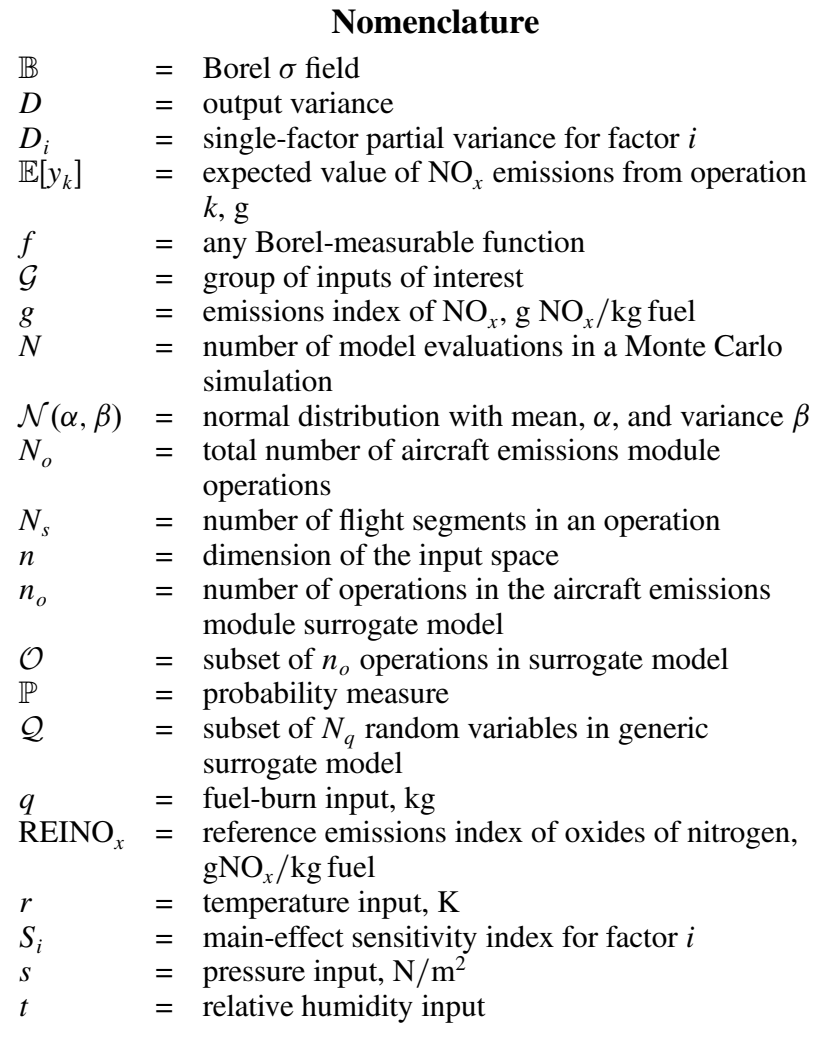

Presented as Paper 2008-5890 at the 12th AIAA/ISSMO Multidisciplinary Analysis and Optimization Conference, Victoria, British Columbia, Canada, 10-12 September 2008; received 24 September 2009; revision received 3 March 2010; accepted for publication 31 March 2010. Copyright () 2010 by the authors. Published by the American Institute of Aeronautics and Astronautics, Inc., with permission. Copies of this paper may be made for personal or internal use, on condition that the copier pay the $\$ 10.00$ per-copy fee to the Copyright Clearance Center, Inc., 222 Rosewood Drive, Danvers, MA 01923; include the code 0001-1452/10 and $\$ 10.00$ in correspondence with the CCC.

*Postdoctoral Associate, Department of Aeronautics and Astronautics; dallaire@mit.edu. Member AIAA.

${ }^{\dagger}$ Associate Professor, Department of Aeronautics and Astronautics; kwillcox@mit.edu. Associate Fellow AIAA.

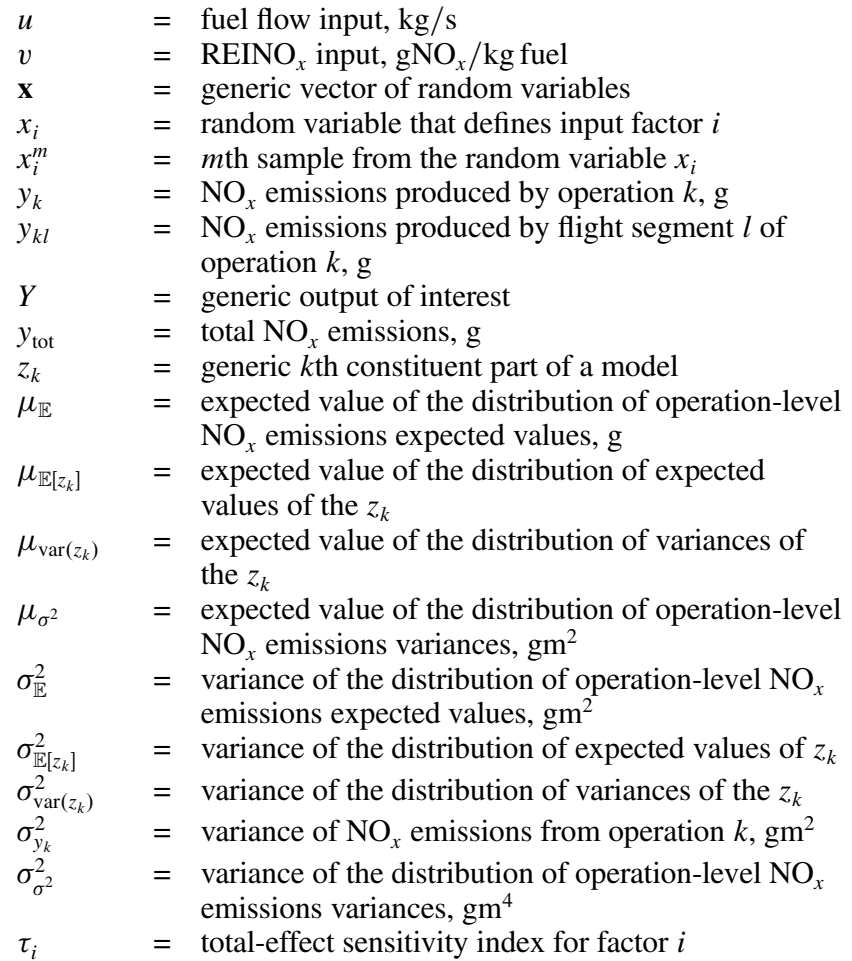

\section{Introduction}

$\mathbf{N}$ UMERICAL simulation models to support decision-making and policy-making processes, while becoming increasingly widespread, typically have uncertainty associated with their inputs, leading to uncertainty in model outputs. Effective application of model results to decision-making and in support of model development require proper characterization, propagation, and analysis of that uncertainty. The process of propagating the uncertainty from inputs to outputs, for example via Monte Carlo simulation, could require many thousands of model evaluations, thus presenting an intractable computational burden. Here, we present a novel surrogate modeling methodology based on invoking the central limit theorem, which is designed specifically for propagating uncertainty from model inputs to model outputs and for performing a global sensitivity 
analysis, which characterizes the contributions of uncertainties in model inputs to output variance, while maintaining the quantitative rigor of the analysis by providing confidence intervals on surrogate predictions.

Our approach is developed for a general class of models where the application of uncertainty propagation and global sensitivity analysis on a full model is computationally impractical, and demonstrated on the specific case of the aircraft emissions model (AEM) of the Aviation Environmental Design Tool (AEDT). The computational models of AEDT are being developed and applied to support aviation environmental policy-making by providing the capability to characterize and quantify interdependencies among aviation-related noise and emissions, impacts on health and welfare, and industry and consumer costs, under different policy, technology, operational, and market scenarios. A key priority is to inform the analyses conducted by these tools with associated uncertainty from the inputs and assumptions used in the analysis process. The scale and complexity of these analyses are immense; for example, a single simulation of a one-year analysis involves over 30 million flight operations with 350 aircraft types and thousands of inputs, analyzed with computationally intensive models spanning airline economics, environmental economics, aircraft operations, aircraft performance and emissions, noise, local air quality, and global climate. Thus, the propagation and analysis of uncertainty in such models with a method such as Monte Carlo simulation, which in some cases can take several thousand model evaluations, is computationally impractical.

Surrogate models that provide substantial computational speedups are therefore crucial to the process of uncertainty analysis in tools such as AEDT. However, quantifying the impacts on the analyses due to exercising a surrogate in place of the full model is essential to producing defensible claims in the context of decision-making. While surrogate modeling methodologies have been successfully applied in many settings, a key challenge here is the derivation of surrogate models, and the associated confidence in uncertainty and sensitivity analyses conducted with the surrogate models, for large-scale complex system models with high-dimensional input spaces.

This paper proposes a systematic method to reduce the complexity and computational cost of a general class of large-scale models in such a way that input uncertainty may still be quantified and analyzed. The method is applied to the AEM, which is designed to estimate global emissions from aviation. Section II presents background on the uncertainty analysis methods employed, and describes the structure of the general class of problems considered. The methodology, described in Sec. III, focuses on the creation of a hierarchical surrogate model for the general model class, by selecting a small subset of inputs to represent the large-scale complex system. These representative inputs form a surrogate model with which an inexpensive computation can be performed in place of the originally expensive computation. In Sec. IV the method is applied to create surrogates for the AEM. We demonstrate how these surrogates can be used for both uncertainty and sensitivity analysis with rigorous confidence intervals on surrogate predictions. Limitations and additional sources of error are discussed in Sec. V, and conclusions are drawn in Sec. VI.

\section{Background}

In the context of numerical simulation tools, uncertainty analysis encompasses the process of characterizing and analyzing the effects of uncertainty in model inputs, with a focus on quantitative assessment of the effects on model outputs and thus on the conclusions drawn from simulation results. Sensitivity analysis studies how variability in model outputs can be apportioned to sources of uncertainty in model factors [1]. To carry out uncertainty and sensitivity analyses for large-scale numerical models requires first an understanding of the purpose of the analyses and the way in which quantitative results will be employed for decision-making. Second, knowledge of the character/structure of the underlying model is important to determine appropriate analysis methods and for an appreciation of the associated computational complexity, which may mandate the use of surrogate models. Background on each of these areas is given in the following subsections.

\section{A. Uncertainty Analysis and Sensitivity Analysis}

A detailed overview of both deterministic and statistical methods for uncertainty and sensitivity analysis of large-scale systems is presented in [1]. Here, we consider two general applications of uncertainty and sensitivity analysis. The first is to support decisionmaking, for which uncertainty analysis should provide the ability to compare various scenarios (e.g., different policies, different input assumptions, etc.) in terms of output means, output variances and other distributional information that may be used to help make a decision. The second application is to help further model development. In this second case, the primary goal is a sensitivity analysis that apportions model output variability to model factors [2-4] to help determine where future research and development efforts should focus.

The computation of model output means, output variances and other distributional information in support of uncertainty analysis for decision-making can be carried out with Monte Carlo simulation. We consider a general model $f(\mathbf{x})$, where $\mathbf{x}=\left[x_{1}, x_{2}, \ldots, x_{n}\right]^{T}$ is the vector of $n$ inputs to the model. If the model inputs are viewed as random variables with some associated probability distribution, then the mean value of the model output can be computed from a Monte Carlo simulation as

$$
\frac{1}{N} \sum_{m=1}^{N} f\left(\mathbf{x}^{m}\right) \rightarrow \mathbb{E}[f(\mathbf{x})] \quad \text { as } N \rightarrow \infty
$$

where $N$ is the number of model evaluations in the Monte Carlo simulation and $\mathbf{x}^{m}=\left[x_{1}^{m}, x_{2}^{m}, \ldots, x_{n}^{m}\right]^{T}$ denotes the $m$ th sample realization of the random vector $\mathbf{x}$. Convergence of the sample mean in Eq. (1) to the expected value of $f(\mathbf{x})$ is guaranteed by the law of large numbers and the convergence rate is $1 / \sqrt{N}$, as given by the central limit theorem [5]. Output variances and other distributional quantities can similarly be computed using Monte Carlo simulation results. The process of computing such quantities requires a large number of model evaluations, which for computationally intensive models is in many cases impractical; hence the need for developing surrogate models for this type of uncertainty analysis.

For model development purposes, global sensitivity analysis is a rigorous method for quantitatively apportioning output variance [2]. The goal of a global sensitivity analysis is shown notionally in Fig. 1 , where the pie represents the variance in a model output, which is then broken out according to factor contributions. The results of a global sensitivity analysis permit a ranking of model factors that can be used in different development settings such as factor prioritization for future research, where the goal is to determine which factors, once fixed will cause the largest reduction in variance, and factor fixing, for which the goal is to identify noninfluential factors that may be fixed without substantially affecting model outputs [].

The process of apportioning output variance across model factors in a global sensitivity analysis can be carried out rigorously by both a Fourier amplitude sensitivity test (FAST) method, and the Sobol' method $[2,3,6,7]$. The FAST method is based on Fourier transforms, while the Sobol' method uses Monte Carlo simulation. Owing to its ease of implementation, the Sobol' method is employed in this work. It should be noted here that other techniques, such as the method of elementary effects due to Morris [8], can approximate the results of the FAST and Sobol' methods, and in the case of the Morris method,

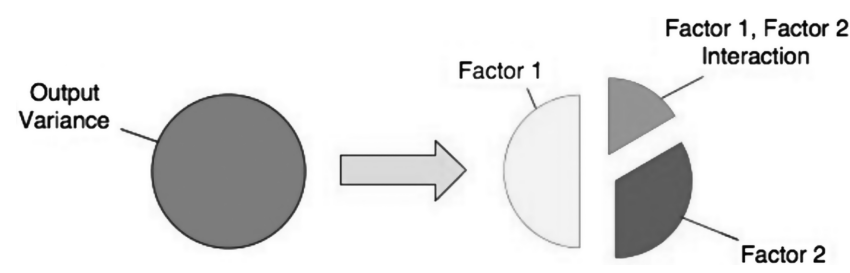

Fig. 1 Apportioning output variance. 
provide a good proxy to the total sensitivity index discussed below [4]. However, in this work we have focused on using rigorous statistical methods on surrogate models rather than on using approximate statistical methods on surrogate models or full models.

The Sobol' method for computing global sensitivity indices is well developed and in wide use in the sensitivity analysis field, particularly by the Joint Research Centre of the European Commission $[2,4,9]$. The method is discussed here in detail because the surrogate modeling methods developed in Sec. III will make use of the formulation. The derivation follows the work of Homma and Saltelli [3].

The Sobol' method is based on the ANOVA high-dimensional model representation (ANOVA-HDMR). A high-dimensional model representation of a function, $f(\mathbf{x})$, can be written as

$$
\begin{aligned}
& f(\mathbf{x})=f_{0}+\sum_{i} f_{i}\left(x_{i}\right)+\sum_{i<j} f_{i j}\left(x_{i}, x_{j}\right)+\cdots \\
& \quad+f_{12, \ldots, n}\left(x_{1}, x_{2}, \ldots, x_{n}\right)
\end{aligned}
$$

where $f_{0}$ is a constant, $f_{i}\left(x_{i}\right)$ is a function of only $x_{i}, f_{i j}\left(x_{i}, x_{j}\right)$ is a function of only $x_{i}$ and $x_{j}$, etc. Without any constraints, the representation of $f(\mathbf{x})$ given by Eq. (2) is not unique; however, it can be made unique by enforcing the constraints

$$
\begin{gathered}
\int_{0}^{1} f_{i_{1}, \ldots, i_{s}}\left(x_{i_{1}}, \ldots, x_{i_{s}}\right) \mathrm{d} x_{\omega}=0, \quad \text { for } \omega=i_{1}, \ldots, i_{s} \\
s=1, \ldots, n
\end{gathered}
$$

where the function $f(\mathbf{x})$, and hence all its components, has been assumed to be integrable. For simplicity of presentation, the inputs to the function in Eq. (3) have been defined on the interval [0,1], but this assumption is not essential to the method. For each $s$, the indices $i_{1}, \ldots, i_{s}$ in Eq. (3) are all sets of $s$ integers such that $1 \leq i_{1}<\cdots<i_{s} \leq n$. Thus, for $s=1$, the constraint (3) applies to all terms $f_{i}$ in Eq. (2), while for $s=2$, the constraint (3) applies to all terms $f_{i j}$ with $i<j$ as in Eq. (2), etc. The application of the constraint (3) makes the HDMR a unique representation of the function $f(\overline{\mathbf{x}})$, referred to as an ANOVA-HDMR. Integration of $f(\mathbf{x})$ over all inputs results in $\int f(\mathbf{x}) \mathrm{d} \mathbf{x}=f_{0}$, which assuming each input $x_{i}$ is a uniform random variable on $[0,1]$, is the mean value of the function $f(\mathbf{x})$.

The constraint given by Eq. (3) also forces the different components of $f(\mathbf{x})$ within the ANOVA-HDMR to be orthogonal. That is, if $\left(i_{1}, \ldots, i_{s}\right) \neq\left(j_{1}, \ldots, j_{l}\right)$, then

$$
\int f_{i_{1}, \ldots, i_{s}}\left(x_{i_{1}}, \ldots, x_{i_{s}}\right) \times f_{j_{1}, \ldots, j_{l}}\left(x_{j_{1}}, \ldots, x_{j_{l}}\right) \mathrm{d} \mathbf{x}=0
$$

since at least one index is not repeated.

Assuming now that $f(\mathbf{x})$ is square integrable, and therefore all components within the ANOVA-HDMR are as well, the variance of $f(\mathbf{x})$ is written as

$$
D=\int f(\mathbf{x})^{2} \mathrm{~d} x-f_{0}^{2}
$$

and partial variances are defined as

$$
D_{i_{1}, \ldots, i_{s}}=\int f_{i_{1}, \ldots, i_{s}}\left(x_{i_{1}}, \ldots, x_{i_{s}}\right)^{2} \mathrm{~d} x_{i_{1}}, \ldots, \mathrm{d} x_{i_{s}}
$$

Given the ANOVA-HDMR for some $f(\mathbf{x})$, we square and then integrate both sides of Eq. (2) and employ the orthogonality constraint to arrive at

$$
\int f(\mathbf{x})^{2} \mathrm{~d} x=f_{0}^{2}+\sum_{i} D_{i}+\sum_{i<j} D_{i j}+\cdots+D_{12, \ldots, n}
$$

which implies

$$
D=\sum_{i} D_{i}+\sum_{i<j} D_{i j}+\cdots+D_{12, \ldots, n}
$$

This is precisely the notion shown in Fig. 1 .
Global sensitivity indices are defined as

$$
S_{i_{1}, \ldots, i_{s}}=\frac{D_{i_{1}, \ldots, i_{s}}}{D}, \quad s=1, \ldots, n
$$

The sum of all global sensitivities of this form for a given function is unity. Global sensitivity indices with only one subscript (e.g., $S_{i}$ ), are called main-effect sensitivities, and those with multiple subscripts (e.g., $S_{i, j}, S_{i, j, k}$, etc.), are called interaction-effect sensitivities. The sum of a factor's main-effect global sensitivity and all interactioneffect sensitivities that involve that factor gives the total-effect sensitivity index, $\tau$, which is defined for input factor $i$ as

$$
\tau_{i}=S_{i}+S_{i, i^{c}}
$$

where $S_{i}$ is the main-effect sensitivity to factor $i$, and $S_{i, i^{c}}$ is the sum of the sensitivity indices of all interaction effects that include factor $i$. Since the sum of all unique sensitivity indices is unity, we have that

$$
\tau_{i}=S_{i}+S_{i, i^{c}}=1-S_{i^{c}}
$$

where $S_{i^{c}}$ is the sum of the sensitivity indices for all main effects and interactions effects that do not involve factor $i$. Since interaction effects will be counted for each factor involved in them, $\Sigma_{i} \tau_{i} \geq 1$.

The total-effect sensitivity indices in Eq. (11) can be computed via Monte Carlo simulation as follows [3], where hat quantities denote estimates of the corresponding true quantities. Here, it should be noted that the computation of the partial variances with Monte Carlo simulation proceeds directly with the function $f(\mathbf{x})$ and does not require explicit knowledge of the functions on the right-hand side of Eq. (2). The estimate of the mean $f_{0}$ is computed as

$$
\hat{f}_{0}=\frac{1}{N} \sum_{m=1}^{N} f\left(\mathbf{x}^{m}\right)
$$

while the estimate of the variance $D$ is

$$
\hat{D}=\frac{1}{N} \sum_{m=1}^{N} f\left(\mathbf{x}^{m}\right)^{2}-\hat{f}_{0}^{2}
$$

The single-factor partial variance is then computed for factor $i$ by resampling all factors except factor $i$ :

$$
\begin{aligned}
\hat{D}_{i} & =\frac{1}{N} \sum_{m=1}^{N} f\left(\left[x_{1}^{m}, \ldots, x_{i}^{m}, \ldots, x_{n}^{m}\right]^{T}\right) f\left(\left[\tilde{x}_{1}^{m}, \ldots, x_{i}^{m}, \ldots, \tilde{x}_{n}^{m}\right]^{T}\right) \\
& -\hat{f}_{0}^{2}, \quad i=1, \ldots, n
\end{aligned}
$$

where $\tilde{x}_{j}^{m}$ denotes a different sample of factor $x_{j}$. The estimate of the variance due to all factors except factor $i$ (which includes the sum of all single-factor and interaction-effect partial variances that do not include factor $i$ ) is denoted as $\hat{D}_{i^{c}}$ and is computed by

$$
\begin{aligned}
\hat{D}_{i^{c}} & =\frac{1}{N} \sum_{m=1}^{N} f\left(\left[x_{1}^{m}, \ldots, x_{i}^{m}, \ldots, x_{n}^{m}\right]^{T}\right) f\left(\left[x_{1}^{m}, \ldots, \tilde{x}_{i}^{m}, \ldots, x_{n}^{m}\right]^{T}\right) \\
& -\hat{f}_{0}^{2}
\end{aligned}
$$

where now just factor $i$ is resampled. Finally, computing $\hat{S}_{i^{c}}=$ $\hat{D}_{i^{c}} / \hat{D}$ and applying Eq. (11), we obtain the desired total-effect sensitivity index.

The main-effect sensitivity indices $S_{i}$ may be used for factor prioritization by ranking inputs according to their main-effect indices, which give the percentage of how much output variability can be expected to be eliminated by fixing a particular input somewhere on its domain. The total-effect sensitivity indices $\tau_{i}$ may be used for factor fixing, since a low total-effect index reveals a given input has a small main effect and also does not take part in substantial interactions among other inputs. For $n$ inputs, the calculation of sensitivity indices requires $(2 n+1)$ Monte Carlo simulations (each with $N$ model evaluations) if both the main-effect and total-effect indices are desired. Thus, like uncertainty analysis for decision-making, 
sensitivity analysis of large-scale models for development purposes will in most situations require surrogate models.

\section{B. Surrogate Modeling}

Surrogate models can be categorized into three different classes: data-fit models, reduced-order models, and hierarchical models [10]. Data-fit models are generated using interpolation or regression of simulation data from the input/output relationships in the highfidelity model $[10,11]$. The primary challenge in adopting this surrogate modeling strategy for large-scale complex system models is the "curse of dimensionality" when the number of inputs to a model is large and design of experiment techniques must be applied with care in order to balance the computational cost of the required simulations with coverage of the input space. Reduced-order models are typically constructed for systems described by partial differential equations or large sets of ordinary differential equations [12]. Derivation of reduced-order models relies on the knowledge of the governing equations and are thus not suitable to systems for which the governing equations are unknown or empirically based. Hierarchical surrogate models, also known as variable-fidelity models, employ simplified mathematical models such as coarser grids in finite element models [13] and models with simplified physics [14-16].

The application of a particular surrogate modeling strategy depends both on what computational tasks are to be performed, and on the underlying structure of the model. In some cases, nothing will be known about a given model, and strategies that perform better in black-box situations, such as data-fit methods should be used. In other cases, everything will be known about the governing equations of a given model, and reduced-order models can be derived using projection-based approaches. In this paper, we consider models of a general form where the outputs of interest are computed as a sum of many constituent parts. As described in the next subsection, this class of models has applications in engineering and logistics systems. For such models, existing surrogate modeling methods cannot be applied. While the structure of the model is known, the input space is too large to use a projection-based model reduction approach or a data-fit method.

\section{Generalized Model Form}

Consider a probability triple $(\mathbb{R}, \mathbb{B}, \mathbb{P})$, where $\mathbb{R}$ is the sample space, here the set of real numbers, $\mathbb{B}$ is the $\sigma$ field (here, the Borel $\sigma$ field), and $\mathbb{P}$ is a probability measure. Also, consider a class of $\mathbb{B}$-measurable functions, $f$. The class of models we consider in this work are of the form

$$
Y=\sum_{k=1}^{N} z_{k}=\sum_{k=1}^{N} f^{k}(\mathbf{x})
$$

where $Y$ is an output of interest computed via a sum over $N$ constituent parts, which are represented by the $z_{k}$ that may themselves be functions of random inputs, $z_{k}=f^{k}(\mathbf{x})$, where $\mathbf{x}=\left[x_{1}, x_{2}, \ldots, x_{n}\right]^{T}$ is a vector of $n$ independent random inputs, and $f^{k} \in f$. Because the $f^{k}$ are all $\mathbb{B}$-measurable, the $z_{k}$ and $Y$ are random variables.

Though the form of Eq. (16) is simple, it is relevant to a broad range of applications. For example, outputs of the form of Eq. (16) are common in systems whose performance is computed over many parts, e.g., emissions or fuel burn summed over mission segments for a transportation system, system failure time estimated as a sum of individual component lifetimes, many aspects of wireless communication, including current fluctuations in tunnel junctions, diversity schemes, and cochannel interference, profit summed over products or store locations, or votes summed over polling locations. In these examples, and in general, it is important to note that the assumption of independence is an aspect of the modeling. Our purpose here is to create surrogates for models of the form given by Eq. (16) so that we may carry out both uncertainty and sensitivity analyses in a reasonable amount of time while maintaining quantitative rigor. If independence is assumed when the full model is exercised, it should be assumed when the surrogate model is used in place of the full model, since the surrogate is attempting to produce estimates of results that would be obtained with the full model. The same sentiment holds when considering the assumption of randomness.

Simpson et al. [17] present a survey of surrogate modeling techniques, along with recommendations for selecting a modeling approach. They find that response surface modeling is appropriate for applications with less than ten input factors, while interpolationbased methods such as kriging may be suitable for up to 50 input factors. For our problems of interest, the number of inputs is typically in the thousands or even millions. Of existing surrogate modeling methods, only neural networks are even remotely possible for such a large number of inputs. However, as discussed in [17], neural networks are computationally expensive to create and are best suited for deterministic problems. More recent advances in surrogate modeling methods, such as the pseudo response surface methodology [18], address some of the computational challenges associated with high-dimensional input spaces by requiring the surrogate to be accurate only in some regions of the design space (e.g., near the Pareto front). However, even with these advances, surrogate modeling for systems with thousands or millions of inputs remains out of reach. In the next section we present a hierarchical surrogate modeling approach that addresses this challenge for models of the form given by Eq. (16).

\section{Surrogate Modeling Methodology with Quantified Confidence Intervals}

Here, we propose a hierarchical surrogate modeling approach targeted at uncertainty analysis and sensitivity analysis applications for the class of models described in the previous section. However, if the uncertainty associated with the use of a surrogate model in place of a full model is not properly quantified, the usefulness of the various uncertainty analyses will be limited. Thus, one of the key objectives of this work is to quantify the effects of using a surrogate model to perform uncertainty and sensitivity analyses. The methods used to achieve this goal for the general class of models given by Eq. (16) are discussed in the following subsections.

\section{A. Hierarchical Surrogate Modeling Approach}

For the class of models given by Eq. (16), a natural representative for building a hierarchical surrogate is a single random variable, $z_{k}$. Our surrogate modeling approach is thus to approximate the output of interest, $Y$, using a subset of the $z_{k}$. For the case of a general model of the form of Eq. (16), if the $z_{k}$ are such that

$$
\max _{c \leq N} \frac{\operatorname{var}\left(z_{c}\right)}{\sum_{k=1}^{N} \operatorname{var}\left(z_{k}\right)} \rightarrow 0 \quad \text { as } N \rightarrow \infty
$$

where $c \in\{1,2, \ldots, N\}$, and $N$ is the number of constituent parts used in the summation given in Eq. (16), then according to the central limit theorem,

$$
Y \stackrel{d}{\rightarrow} \mathcal{N}\left(\sum_{k=1}^{N} \mathbb{E}\left[z_{k}\right], \sum_{k=1}^{N} \operatorname{var}\left(z_{k}\right)\right) \quad \text { as } N \rightarrow \infty
$$

where the convergence is in distribution, $\mathcal{N}(\alpha, \beta)$ is a normal distribution with mean $\alpha$ and variance $\beta$, and the constraint given by Eq. (17) is referred to as the Lyapunov condition [19]. Though Eq. (18) states that the convergence to a normal distribution occurs as $N \rightarrow \infty$, it is common in statistical practice to assume $Y$ may be appropriately modeled with a normal distribution when $N \geq 30$ [20]. The constraint given by Eq. (17) can be met, for example, by any set of $z_{k}$ such that $\operatorname{var}\left(z_{k}\right)<\infty$ for $k=1,2, \ldots, N$ and $\Sigma_{k=1}^{N} \operatorname{var}\left(z_{k}\right)$ is unbounded as $N \rightarrow \infty$. Although independence was used here to invoke the central limit theorem, there are other methods for invoking the central limit theorem, such as $m$ dependence [21], for situations in which the independence condition is not met. The application of the central limit theorem to the general model given in Eq. (16) for both 
uncertainty and sensitivity analyses is discussed in the following section.

\section{B. Surrogate Modeling for Decision-Making Uncertainty Analysis}

As given by Eq. (18), the output of interest, $Y$, is normally distributed. To estimate the distribution of $Y$ with a surrogate model, only estimates of $\Sigma_{k=1}^{N} \mathbb{E}\left[z_{k}\right]$ and $\Sigma_{k=1}^{N} \operatorname{var}\left(z_{k}\right)$ are required. We may estimate these quantities by noting that if we were to compute $\mathbb{E}\left[z_{k}\right]$ for every constituent part $z_{k}$, we could view the resulting set of expected values as representing a set of $N$ samples drawn from some distribution. Thus, the expected value of some $z_{k}$ can be considered as a sample from a random variable, and can be estimated using a subset of random variables chosen from the full set. We denote by $\mathcal{Q}$ the subset of $N_{q}$ random variables chosen randomly from the full set of $N$ random variables. Then using the law of large numbers, $\Sigma_{k=1}^{N} \mathbb{E}\left[z_{k}\right]$ is estimated as $N\left(1 / N_{q}\right) \Sigma_{k \in \mathcal{Q}} \mathbb{E}\left[z_{k}\right]$, since

$$
\begin{aligned}
N & \frac{1}{N_{q}} \sum_{k \in \mathcal{Q}} \mathbb{E}\left[z_{k}\right] \rightarrow N \mathbb{E}\left[\mathbb{E}\left[z_{k}\right]\right]=N \frac{1}{N} \sum_{k=1}^{N} \mathbb{E}\left[z_{k}\right] \\
= & \sum_{k=1}^{N} \mathbb{E}\left[z_{k}\right] \text { as } N_{q} \rightarrow N
\end{aligned}
$$

For the sum of the variances of the $z_{k}$ in Eq. (18), a similar method is followed to derive an analogous expression for the variance estimate of $Y$. Thus, the surrogate model estimate of the output distribution using the subset $\mathcal{Q}$ of $N_{q}$ random variables to represent the full $N$ random variables is given by

$$
\hat{Y} \sim \mathcal{N}\left(\frac{N}{N_{q}} \sum_{k \in \mathcal{Q}} \mathbb{E}\left[z_{k}\right], \frac{N}{N_{q}} \sum_{k \in \mathcal{Q}} \operatorname{var}\left(z_{k}\right)\right)
$$

where $\hat{Y}$ is a random variable that is an estimate of the random variable $Y$.

In (20), the terms $\left(1 / N_{q}\right) \Sigma_{k \in \mathcal{Q}} \mathbb{E}\left[z_{k}\right]$ and $\left(1 / N_{q}\right) \Sigma_{k \in \mathcal{Q}} \operatorname{var}\left(z_{k}\right)$, are sample means of the distributions of expected values of the $z_{k}$ and of the variances of the $z_{k}$, respectively. According to the central limit theorem, these sample means have the following normal distributions:

$$
\begin{gathered}
\frac{1}{N_{q}} \sum_{k \in \mathcal{Q}} \mathbb{E}\left[z_{k}\right] \sim \mathcal{N}\left(\mu_{\mathbb{E}\left[z_{k}\right]}, \frac{N-N_{q}}{N-1} \sigma_{\mathbb{E}\left[z_{k}\right]}^{2} / N_{q}\right) \\
\frac{1}{N_{q}} \sum_{k \in \mathcal{Q}} \operatorname{var}\left(z_{k}\right) \sim \mathcal{N}\left(\mu_{\operatorname{var}\left(z_{k}\right)}, \frac{N-N_{q}}{N-1} \sigma_{\operatorname{var}\left(z_{k}\right)}^{2} / N_{q}\right)
\end{gathered}
$$

where $\mu_{\mathbb{E}\left[z_{k}\right]}$ is the expected value of the distribution of expected values of the $z_{k}, \sigma_{\mathbb{E}\left[z_{k}\right]}^{2}$ is the variance of the distribution of expected values of $z_{k}, \mu_{\operatorname{var}\left(z_{k}\right)}$ is the expected value of the distribution of variances of the $z_{k}$, and $\sigma_{\operatorname{var}\left(z_{k}\right)}^{2}$ is the variance of the distribution of variances of the $z_{k}$. The $\left(N-N_{q}\right) /(N-1)$ terms are finite population correction factors that must be applied since $N$ is finite and the sampling of $N_{q}$ random variables from $N$ total random variables is done without replacement [22].

As noted in Sec. II, a key outcome of an uncertainty analysis intended to support decision-making is the ability to compare such quantities as output means and variances. These quantities cannot be computed exactly using a surrogate model; however, confidence intervals for these quantities can be rigorously computed since, as shown in the analysis above, the parameters are normally distributed. The confidence intervals for the mean and variance of the output $Y$ can be constructed from

$$
\begin{aligned}
& \frac{N}{N_{q}} \sum_{k \in \mathcal{Q}} \mathbb{E}\left[z_{k}\right]-Z_{\alpha / 2} \sqrt{\frac{N-N_{q}}{N-1} \frac{N^{2}}{N_{q}} \sigma_{\mathbb{E}\left[z_{k}\right]}^{2}}<\mathbb{E}[Y]<\frac{N}{N_{q}} \sum_{k \in \mathcal{Q}} \mathbb{E}\left[z_{k}\right] \\
& \quad+Z_{\alpha / 2} \sqrt{\frac{N-N_{q}}{N-1} \frac{N^{2}}{N_{q}} \sigma_{\mathbb{E}\left[z_{k}\right]}^{2}}
\end{aligned}
$$

and

$$
\begin{gathered}
\frac{N}{N_{q}} \sum_{k \in \mathcal{Q}} \operatorname{var}\left(z_{k}\right)-Z_{\alpha / 2} \sqrt{\frac{N-N_{q}}{N-1} \frac{N^{2}}{N_{q}} \sigma_{\operatorname{var}\left(z_{k}\right)}^{2}}<\operatorname{var}(Y) \\
<\frac{N}{N_{q}} \sum_{k \in \mathcal{Q}} \operatorname{var}\left(z_{k}\right)+Z_{\alpha / 2} \sqrt{\frac{N-N_{q}}{N-1} \frac{N^{2}}{N_{q}} \sigma_{\operatorname{var}\left(z_{k}\right)}^{2}}
\end{gathered}
$$

where $Z_{\alpha / 2}$ is the value of the inverse cumulative distribution function of a standard normal random variable evaluated at $(1-\alpha / 2)$, where $\alpha$ sets the level of confidence [23]. A typical value of $Z_{\alpha / 2}$ is 1.96 , which corresponds to a $95 \%$ confidence interval. In practice, constructing these confidence intervals requires estimating the variance of the distribution of the expected values of the $z_{k}, \sigma_{\mathbb{E}\left[z_{k}\right]}^{2}$, and the variance of the distribution of the variances of the $z_{k}, \sigma_{\operatorname{var}\left(z_{k}\right)}^{2}$. We estimate these parameters using the sample variance for each, which are calculated from

$$
\begin{gathered}
\hat{\sigma}_{\mathbb{E}\left[z_{k}\right]}^{2}=\frac{1}{N_{q}-1} \sum_{k=1}^{N_{q}}\left(\mathbb{E}\left[z_{k}\right]-\overline{\mathbb{E}\left[z_{k}\right]}\right)^{2} \\
\hat{\sigma}_{\operatorname{var}\left(z_{k}\right)}^{2}=\frac{1}{N_{q}-1} \sum_{k=1}^{N_{q}}\left(\operatorname{var}\left(z_{k}\right)-\overline{\operatorname{var}\left(z_{k}\right)}\right)^{2}
\end{gathered}
$$

where $\hat{\sigma}_{\mathbb{E}\left[z_{k}\right]}^{2}$ and $\hat{\sigma}_{\operatorname{var}\left(z_{k}\right)}^{2}$ are the sample variances of $\sigma_{\mathbb{E}\left[z_{k}\right]}^{2}$ and $\sigma_{\operatorname{var}\left(z_{k}\right)}^{2}$, respectively, and $\overline{\mathbb{E}\left[z_{k}\right]}$ and $\overline{\operatorname{var}\left(z_{k}\right)}$ are the sample means of the distributions of the expected values and the variances of the $z_{k}$, respectively. The estimates, $\hat{\sigma}_{\mathbb{E}\left[z_{k}\right]}^{2}$ and $\hat{\sigma}_{\operatorname{var}\left(z_{k}\right)}^{2}$, are then used in Eqs. (22) and (23). These intervals also require the estimation of expected values of the random variables $z_{k}, \mathbb{E}\left[z_{k}\right]$, and variances, $\operatorname{var}\left(z_{k}\right)$. These parameters, as will be discussed in Sec. IV, are estimated from a Monte Carlo simulation with a large number of model evaluations, and thus uncertainty associated with these estimates is neglected.

As can be seen from Eq. (22), as $N_{q}$ approaches $N$, the confidence interval around $\mathbb{E}[Y]$ narrows, eventually becoming a single point when $N_{q}=N$. Thus, there is a tradeoff between how many random variables are considered in the surrogate model and the tightness of the confidence intervals for the mean and variance of the output $Y$.

\section{Surrogate Modeling for Model Development Sensitivity Analysis}

The ANOVA-HDMR for a random variable $z_{k}$ given by $f^{k}(\mathbf{x})$ may be written as

$$
\begin{aligned}
z_{k} & =f_{0}^{k}+\sum_{i} f_{i}^{k}\left(x_{i}\right)+\sum_{i<j} f_{i j}^{k}\left(x_{i}, x_{j}\right)+\cdots \\
& +f_{12, \ldots, n}^{k}\left(x_{1}, x_{2}, \ldots, x_{n}\right)
\end{aligned}
$$

which can be squared and integrated, as was done to arrive at Eq. (7), giving

$$
\operatorname{var}\left(z_{k}\right):=\sum_{i} D_{i}^{k}+\sum_{i<j} D_{i j}^{k}+\cdots+D_{12, \ldots, n}^{k}
$$

where $D_{i}^{k}$ is the partial variance of $z_{k}$ due to input $x_{i}$ and the rest of the terms represent partial variances of $z_{k}$ due to various levels of interactions between the components of $\mathbf{x}$. Summing over all the $z_{k}$ gives

$$
\begin{aligned}
Y= & \sum_{k=1}^{N} f_{0}^{k}+\sum_{k=1}^{N} \sum_{i} f_{i}^{k}\left(x_{i}\right)+\sum_{k=1}^{N} \sum_{i<j} f_{i j}^{k}\left(x_{i}, x_{j}\right)+\cdots \\
& +\sum_{k=1}^{N} f_{12, \ldots, n}^{k}\left(x_{1}, x_{2}, \ldots, x_{n}\right)
\end{aligned}
$$

At this point we may proceed as we did in Sec. II.A and estimate totaland main-effect sensitivity indices for each $x_{i}$. However, the models 


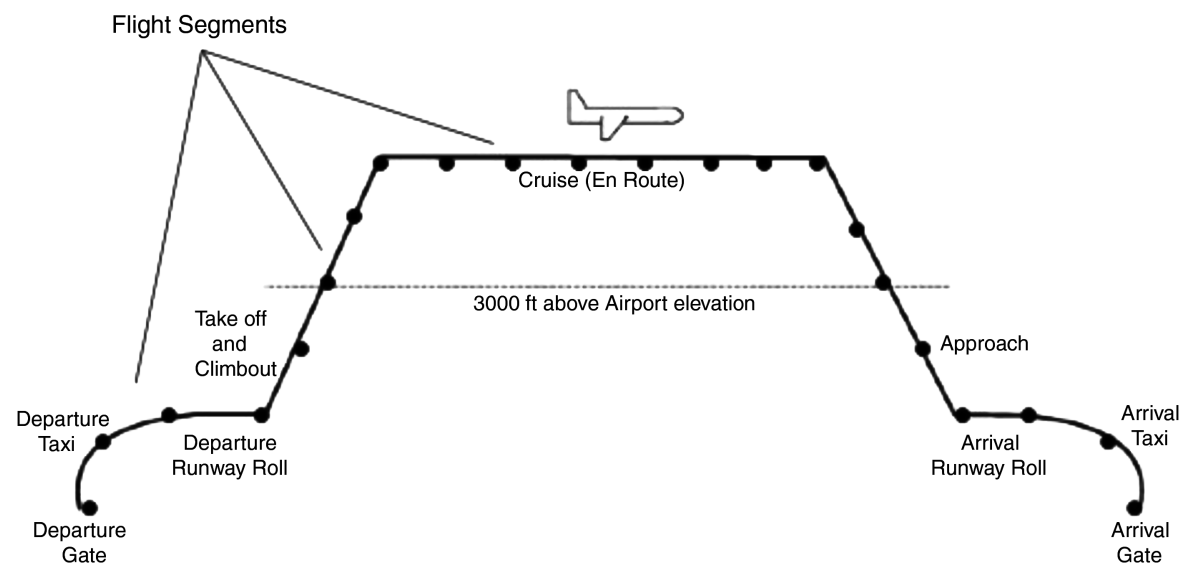

Fig. 2 AEM segmentation of an aircraft operation; adapted from [25].

of the form given by Eq. (16) may contain millions of inputs, rendering computation of sensitivity indices for each input impractical and of limited use. For such models, it is typical that these inputs comprise just a few distinct physical quantities. For example, for the emissions model analyzed in the next section, the inputs consist of six physical quantities (fuel burn, temperature, pressure, relative humidity, fuel flow, and reference emission index) which are defined for each flight segment of each operation in a set of aircraft flights. A sensitivity analysis could consider the sensitivities of each of these independently sampled inputs individually, resulting in millions of sensitivity indices. From a practical standpoint, we are more interested in determining the sensitivity of model outputs to groups of inputs. For example, for the nitrous oxide $\left(\mathrm{NO}_{x}\right)$ emissions for an aircraft operation, we might wish to compute the contribution to variance of all fuel-burn inputs for that operation, where each input is sampled independently across flight segments in the operation. Alternatively, for the total $\mathrm{NO}_{x}$ emissions summed over a set of operations, we might wish to compute the contribution to variance of all fuel-burn inputs for those operations, where again each input is sampled independently across all flight segments. In this section, we present the extension of the global sensitivity analysis methodology to handle such cases for the class of general models given by Eq. (16). Further, application of the central limit theorem, which is permissible given the additive nature of the models and ANOVA-HDMR, enables the calculation of confidence intervals around sensitivity indices computed using the surrogate models in place of the full models.

Consider a case in which we have two physical quantities (type 1 and type 2, e.g., pressure and temperature) within $\mathbf{x}$ that we wish to group. We define the set of physical quantities of type 1 as

$$
\mathcal{G}_{1}=\left\{x_{i} \mid x_{i} \text { are of type } 1\right\}
$$

and the set of physical quantities of type 2 as

$$
\mathcal{G}_{2}=\left\{x_{i} \mid x_{i} \text { are of type } 2\right\}
$$

An ANOVA-HDMR can then be written for $Y$ in terms of the groups $\mathcal{G}_{1}$ and $\mathcal{G}_{2}$ and their interaction rather than the individual components of $\mathbf{x}$. The variance of $Y$ can then be written as

$$
\operatorname{var}(Y):=D=D_{\mathcal{G}_{1}}+D_{\mathcal{G}_{2}}+D_{\mathcal{G}_{1} \mathcal{G}_{2}}
$$

where $D_{\mathcal{G}_{1}}$ is the variance of $Y$ due to the elements of $\mathcal{G}_{1}$ and their interactions, $D_{\mathcal{G}_{2}}$ is the variance of $Y$ due to the elements of $\mathcal{G}_{2}$ and their interactions, and $D_{\mathcal{G}_{1} \mathcal{G}_{2}}$ is the variance of $Y$ due to the interactions involving elements from both $\mathcal{G}_{1}$ and $\mathcal{G}_{2}$. A global sensitivity analysis may then be carried out to compute total-effect and main-effect sensitivity indices for the groups. For $\mathcal{G}_{1}$, the totaleffect sensitivity index can be computed as

$$
\tau_{\mathcal{G}_{1}}=1-\frac{D_{\mathcal{G}_{1}^{c}}}{D}
$$

where $D_{\mathcal{G}_{1}^{c}}$ is the sum of the variances due to all main-effect terms that do not involve elements of $\mathcal{G}_{1}$, which here is just $D_{\mathcal{G}_{2}}$. The maineffect sensitivity index can be computed as

$$
S_{\mathcal{G}_{1}}=\frac{D_{\mathcal{G}_{1}}}{D}
$$

Similar expressions may be written for $\mathcal{G}_{2}$.

For the class of models with which we are concerned, which consist of a summation of $N$ constituent parts as in Eq. (16), it is typical that particular physical inputs be defined on each part. Thus, the groups in this case will each have $N$ elements. As will be shown in Sec. IV, computation of the sensitivity indices given by Eqs. (30) and (31) can proceed by performing global sensitivity analyses on the constituent parts of the model. This enables the creation of hierarchical surrogate models for global sensitivity analysis in the form of subsets of constituent parts much like the surrogate models constructed in Sec. III.B. Confidence intervals may be derived for the sensitivity indices computed using these surrogates, the formulation of which is given in the following section.

\section{AEM Application and Results}

In this section, our approach is applied to the example of estimating emissions of aircraft flights. We first describe the model and then present surrogate models for decision-making uncertainty analysis and model development sensitivity analysis. This is followed by results from using the surrogate models in place of the full model for a typical emissions analysis.

\section{A. Aircraft Emissions Model}

The AEM is used to calculate emissions inventories of such pollutants as $\mathrm{CO}_{2}, \mathrm{CO}, \mathrm{NO}_{x}, \mathrm{SO}_{x}$, and many others. The calculation is done on an operation-by-operation basis, and the emissions computed for each operation in a given scenario are then aggregated to produce an emissions inventory. An operation is in turn simulated on a flight segment-by-segment basis as shown in Fig. 2, where emissions are calculated for each segment of the operation and then aggregated to produce the total emissions of the operation.

The AEM inputs considered in our analyses are shown in Table 1, where each input is defined for each segment of each operation. We consider the emissions resulting from a total of $N_{o}$ operations, each consisting of $N_{s}$ flight segments. Thus, the total number of inputs is given by $n=6 N_{o} N_{s}$. Table 1 also shows the probability density functions that are defined for each input on a segment-by-segment basis. These density functions were arrived at through previous studies and expert opinions of the Partnership for Air Transportation Noise and Emissions Reduction (PARTNER). The samples from the density functions are applied as multipliers to default values of the

${ }^{\ddagger}$ Data available online at http://web.mit.edu/aeroastro/partner/index.html [retrieved January 2009]. 
Table 1 AEM inputs and their probability density functions (all inputs are applied as multipliers to nominal input values)

\begin{tabular}{lllc}
\hline \hline Input variable & Input quantity & Distribution type & Defining values \\
\hline$x_{1}:=q$ & Fuel burn & Uniform & {$[0.95,1.05]$} \\
$x_{2}:=r$ & Temperature & Triangular & {$[0.89,1.00,1.11]$} \\
$x_{3}:=s$ & Pressure & Triangular & {$[0.97,1.00,1.03]$} \\
$x_{4}:=t$ & Relative humidity & Triangular & {$[0.82,1.00,1.17]$} \\
$x_{5}:=u$ & Fuel flow & Uniform & {$[0.95,1.05]$} \\
$x_{6}:=v$ & REINO $_{x}$ & Triangular & {$[0.76,1.00,1.24]$} \\
\hline
\end{tabular}

various inputs that are specific to aircraft type, engine type, and geographic location. For triangular distributions, the defining values are the minimum, mode, and maximum values. For uniform distributions, the defining values are the minimum and the maximum.

The outputs of the AEM (global emissions of $\mathrm{NO}_{x}, \mathrm{CO}, \mathrm{CO}_{2}$, etc.) are all computed in a similar manner, and thus the modeling methodology is developed here only for the $\mathrm{NO}_{x}$ output. Other outputs are treated in an analogous way. The $\mathrm{NO}_{x}$ produced for operation $k, y_{k}$, is calculated as

$$
y_{k}=\sum_{l=1}^{N_{s}} q_{k l} g_{k l}\left(r_{k l}, s_{k l}, t_{k l}, u_{k l}, v_{k l}\right)
$$

where $q_{k l}$ is the fuel burn on segment $l$ of operation $k$, and $g_{k l}\left(r_{k l}, s_{k l}, t_{k l}, u_{k l}, v_{k l}\right)$ is the emissions index of $\mathrm{NO}_{x}\left(\mathrm{EINO}_{x}\right)$ on segment $l$ of operation $k$, which is calculated using Boeing method 2 [24], with the inputs defined in Table 1, specifically the temperature, pressure, relative humidity, fuel flow, and reference emissions index of $\mathrm{NO}_{x}\left(\mathrm{REINO}_{x}\right)$ for the given segment. The Boeing Method 2 is an empirical method for correcting reference emission indices for flight conditions using fuel flow and atmospheric conditions. The total $\mathrm{NO}_{x}$ output for a set of $N_{o}$ operations is then calculated as

$$
y_{\text {tot }}=\sum_{k=1}^{N_{o}} y_{k}=\sum_{k=1}^{N_{o}} \sum_{l=1}^{N_{s}} q_{k l} g_{k l}\left(r_{k l}, s_{k l}, t_{k l}, u_{k l}, v_{k l}\right)
$$

Since the functions within the AEM are all continuous, and each input of the AEM is a random variable, each output of the AEM is also a random variable. Thus, the total $\mathrm{NO}_{x}$ output, $y_{\text {tot }}$, can be thought of as a random sample from the random variable $Y_{\text {tot }}$, for which confidence intervals and sensitivity indices are desired.

Equation (33) reveals the structure of the AEM once it has been decomposed by operations. Given that computations are performed separately on each operation within the AEM, it has the general form of Eq. (16), where the $z_{k} \equiv y_{k}$,

$$
f^{k} \equiv \sum_{l=1}^{N_{s}} q_{k l} g_{k l}\left(r_{k l}, s_{k l}, t_{k l}, u_{k l}, v_{k l}\right)
$$

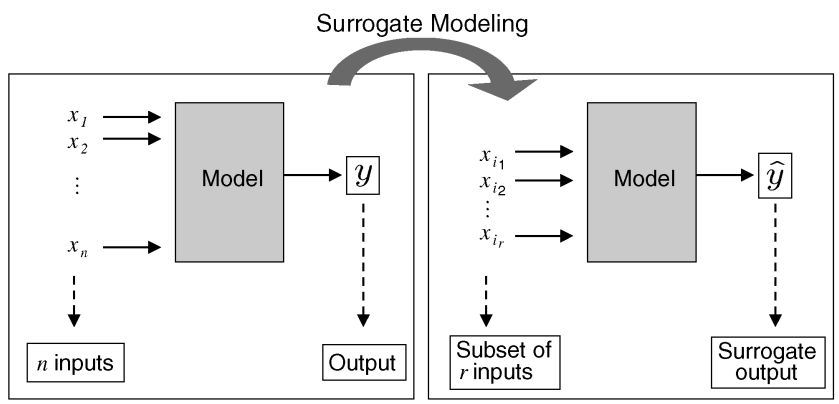

Fig. 3 The hierarchical surrogate modeling approach achieves a reduction in computational complexity through a reduction of the input space. For the AEM, this amounts to selecting a subset of $\boldsymbol{r}$ operations, denoted by the subscripts, $i_{1}, \ldots, i_{r}$, over which to estimate the total emissions. and $\mathbf{x} \equiv\left(q_{k l}, r_{k l}, s_{k l}, t_{k l}, u_{k l}, v_{k l}\right)$. Our surrogate modeling approach thus amounts to approximating the output of interest, total $\mathrm{NO}_{x}$ emissions, using a subset of flight operations. This is illustrated in Fig. 3 , where it can been seen that the surrogate modeling approach is based on reducing the dimension of the input space.

Since the functions within the AEM are all continuous, and each input of the AEM is a random variable, each single operation output of the AEM is also a random variable. These random variables are independent and satisfy the Lyapunov condition [19]; therefore, the AEM is a member of the general class of models described in Sec. II.C and the results presented in Sec. III are applicable. Here, it is noted that, owing to such circumstances as aircraft operating on similar routes, certain operations in the real-world would encounter similar environmental factors, such as temperature, pressure, and humidity, thus causing some dependence in how those factors enter into emissions estimates, which would lead to some dependence in the outputs of these operations. However, the AEM does not currently include these factor dependencies, which implies that the operation-level outputs of the AEM are completely independent of each other. Thus, the central limit theorem may be invoked.

\section{B. AEM Surrogate for Decision-Making Uncertainty Analysis}

According to the central limit theorem, the output distribution of total $\mathrm{NO}_{x}$ emissions, $y_{\text {tot }}$, is normally distributed since

$$
y_{\mathrm{tot}}=\sum_{k=1}^{N_{o}} y_{k} \stackrel{d}{\rightarrow} \mathcal{N}\left(\sum_{k=1}^{N_{o}} \mathbb{E}\left[y_{k}\right], \sum_{k=1}^{N_{o}} \sigma_{y_{k}}^{2}\right) \quad \text { as } N_{o} \rightarrow \infty
$$

where the convergence is in distribution, and $\mathcal{N}(\alpha, \beta)$ is a normal distribution with mean $\alpha$ and variance $\beta$. Typical analyses involving the AEM calculate emissions inventories for representative days of operations, for which $N_{o} \approx 70,000$, and $1 \mathrm{yr}$ of operations, for which $N_{o} \approx 30,000,000$. As noted in Sec. II.C, to assume normality, $N_{o}$ should be greater than about 30 , and thus the number of samples is much greater than required.

To estimate the distribution of $y_{\text {tot }}$ with a surrogate model, only estimates of $\Sigma_{k=1}^{N_{o}} \mathbb{E}\left[y_{k}\right]$ and $\Sigma_{k=1}^{N_{o}} \sigma_{y_{k}}^{2}$ are required. As shown in Sec. III, we may generate a surrogate model estimate of the the total $\mathrm{NO}_{x}$ output distribution using a subset $\mathcal{O}$ of $n_{o}$ operations to represent the full $N_{o}$ operations as

$$
\hat{y}_{\text {tot }} \sim \mathcal{N}\left(\frac{N_{o}}{n_{o}} \sum_{k \in \mathcal{O}} \mathbb{E}\left[y_{k}\right], \frac{N_{o}}{n_{o}} \sum_{k \in \mathcal{O}} \sigma_{y_{k}}^{2}\right)
$$

In Eq. ( $\underline{35})$, the terms $\left(1 / n_{o}\right) \Sigma_{k \in \mathcal{O}} \mathbb{E}\left[y_{k}\right]$ and $\left(1 / n_{o}\right) \Sigma_{k \in \mathcal{O}} \sigma_{y_{k}}^{2}$, are sample means of the distributions of expected values of $y_{k}$ and of the variances of the $y_{k}$, respectively. These sample means then have the following normal distributions:

$$
\begin{aligned}
& \frac{1}{n_{o}} \sum_{k \in \mathcal{O}} \mathbb{E}\left[y_{k}\right] \sim \mathcal{N}\left(\mu_{\mathbb{E}}, \frac{N_{o}-n_{o}}{N_{o}-1} \sigma_{\mathbb{E}}^{2} / n_{o}\right) \\
& \frac{1}{n_{o}} \sum_{k \in \mathcal{O}} \sigma_{y_{k}}^{2} \sim \mathcal{N}\left(\mu_{\sigma^{2}}, \frac{N_{o}-n_{o}}{N_{o}-1} \sigma_{\sigma^{2}}^{2} / n_{o}\right)
\end{aligned}
$$

where $\mu_{\mathbb{E}}$ is the expected value of the distribution of expected values of the $y_{k}, \sigma_{\mathbb{E}}^{2}$ is the variance of the distribution of expected values of $y_{k}, \mu_{\sigma^{2}}$ is the expected value of the distribution of variances of the $y_{k}$, and $\sigma_{\sigma^{2}}^{2}$ is the variance of the distribution of variances of the $y_{k}$.

As given in Sec. III, confidence intervals for the mean and variance of total $\mathrm{NO}_{x}$ emissions can be constructed from

$$
\begin{aligned}
& \frac{N_{o}}{n_{o}} \sum_{k \in \mathcal{O}} \mathbb{E}\left[y_{k}\right]-Z_{\alpha / 2} \sqrt{\frac{N_{o}-n_{o}}{N_{o}-1} \frac{N_{o}^{2}}{n_{o}} \sigma_{\mathbb{E}}^{2}}<\mathbb{E}\left[y_{\mathrm{tot}}\right]<\frac{N_{o}}{n_{o}} \sum_{k \in \mathcal{O}} \mathbb{E}\left[y_{k}\right] \\
& +Z_{\alpha / 2} \sqrt{\frac{N_{o}-n_{o}}{N_{o}-1} \frac{N_{o}^{2}}{n_{o}} \sigma_{\mathbb{E}}^{2}}
\end{aligned}
$$




$$
\begin{aligned}
& \frac{N_{o}}{n_{o}} \sum_{k \in \mathcal{O}} \sigma_{y_{k}}^{2}-Z_{\alpha / 2} \sqrt{\frac{N_{o}-n_{o}}{N_{o}-1} \frac{N_{o}^{2}}{n_{o}} \sigma_{\sigma^{2}}^{2}}<\operatorname{var}\left(y_{\mathrm{tot}}\right)<\frac{N_{o}}{n_{o}} \sum_{k \in \mathcal{O}} \sigma_{y_{k}}^{2} \\
& \quad+Z_{\alpha / 2} \sqrt{\frac{N_{o}-n_{o}}{N_{o}-1} \frac{N_{o}^{2}}{n_{o}} \sigma_{\sigma^{2}}^{2}}
\end{aligned}
$$

As noted in Sec. III, construction of these confidence intervals requires estimating the variance of the distribution of the expected values of the $y_{k}, \sigma_{\mathbb{E}}^{2}$, and the variance of the distribution of the variances of the $y_{k}, \sigma_{\sigma^{2}}^{2}$. We estimate these parameters using the sample variance for each, which are calculated from

$$
\begin{gathered}
\hat{\sigma}_{\mathbb{E}}^{2}=\frac{1}{n_{o}-1} \sum_{k=1}^{n_{o}}\left(\mathbb{E}\left[y_{k}\right]-\overline{\mathbb{E}\left[y_{k}\right]}\right)^{2} \\
\hat{\sigma}_{\sigma^{2}}^{2}=\frac{1}{n_{o}-1} \sum_{k=1}^{n_{o}}\left(\sigma_{y_{k}}^{2}-\bar{\sigma}_{y_{k}}^{2}\right)^{2}
\end{gathered}
$$

where $\hat{\sigma}_{\mathbb{E}}^{2}$ and $\hat{\sigma}_{\sigma^{2}}^{2}$ are the sample variances of $\sigma_{\mathbb{E}}^{2}$ and $\sigma_{\sigma^{2}}^{2}$, respectively, and $\overline{\mathbb{E}}\left[y_{k}\right]$ and $\bar{\sigma}_{y_{k}}^{2}$ are the sample means of the distributions of the expected values and the variances of the $y_{k}$, respectively. The estimates, $\hat{\sigma}_{\mathbb{E}}^{2}$ and $\hat{\sigma}_{\sigma^{2}}^{2}$, are then used in Eqs. (37) and (38). As will be shown in Sec. IV.D, $n_{o}$ is sufficiently large to neglect the uncertainty associated with these estimates. These intervals also require the estimation of operation-level expected values $\mathbb{E}\left[y_{k}\right]$ and variances $\sigma_{y_{k}}^{2}$ of $\mathrm{NO}_{x}$ emissions. These parameters, as will be discussed in Sec. IV.D, are estimated from a Monte Carlo simulation with a large number of model evaluations, and thus uncertainty associated with these estimates is also neglected.

As can be seen from Eq. (37), as $n_{o}$ approaches $N_{o}$, the confidence interval around $\mathbb{E}\left[y_{\text {tot }}\right]$ narrows, eventually becoming a single point when $n_{o}=N_{o}$. Thus, as mentioned in Sec. III, there is a tradeoff between how many operations are analyzed in the surrogate model, and the tightness of the confidence intervals for the mean and variance of the total $\mathrm{NO}_{x}$. This tradeoff is discussed further in Secs. IV.D and VI. Results from applying this method to construct confidence intervals for the mean and variance of the AEM NO output are presented in Sec. IV.D.

\section{AEM Surrogate for Model Development Sensitivity Analysis}

Consider the ANOVA-HDMR for the calculation of the $\mathrm{NO}_{x}$ emissions from a single operation, $l$ :

$$
\begin{aligned}
y_{k} & =f_{0, k}+\sum_{l=1}^{N_{s}} f_{q_{k l}}\left(q_{k l}\right)+\sum_{l=1}^{N_{s}} f_{r_{k l}}\left(r_{k l}\right)+\sum_{l=1}^{N_{s}} f_{s_{k l}}\left(s_{k l}\right) \\
+ & \sum_{l=1}^{N_{s}} f_{t_{k l}}\left(t_{k l}\right)+\sum_{l=1}^{N_{s}} f_{u_{k l}}\left(u_{k l}\right)+\sum_{l=1}^{N_{s}} f_{v_{k l}}\left(v_{k l}\right) \\
& + \text { interaction terms }
\end{aligned}
$$

where we use the input variable notation defined in Table 1 . The term $\Sigma_{l=1}^{N_{s}} f_{q_{k l}}\left(q_{k l}\right)$ is the sum of all the single-factor functions of factor $q_{k l}$; that is, the functions that depend only on the segment fuel-burn inputs. The second summation is over those functions that depend only on the segment temperatures $r_{k l}$, and so on for the other summations. Here, as in Eq. (32), $N_{s}$ segments have been assumed for operation $k$.

Since the goal is to compute sensitivities for inputs grouped across flight segments, we define $q_{k}=\left\{q_{k l}\right\}_{l=1}^{N_{s}}$ to be the set of fuel-burn segment inputs for operation $k$. Define $r_{k}, s_{k}, t_{k}, u_{k}$, and $v_{k}$ similarly for the other input quantities. Each summation in Eq. (41) can then be written as

$$
f_{q_{k}}=\sum_{l=1}^{N_{s}} f_{q_{k l}}\left(q_{k l}\right)
$$

with analogous expressions defining $f_{r_{k}}$, etc. Then Eq. (41) is written as $y_{k}=f_{0, k}+f_{q_{k}}+f_{r_{k}}+f_{s_{k}}+f_{t_{k}}+f_{u_{k}}+f_{v_{k}}+$ interaction terms

Squaring and integrating Eq. (3), as was done to arrive at Eq. (7), gives

$$
\begin{aligned}
\operatorname{var}\left(y_{k}\right):= & D_{y_{k}}=D_{q_{k}}+D_{r_{k}}+D_{s_{k}}+D_{t_{k}}+D_{u_{k}}+D_{v_{k}} \\
& + \text { interaction partial variances }
\end{aligned}
$$

where $D_{q_{k}}$ is the partial variance due to all fuel-burn inputs, and so on for the other inputs.

Similarly, the AEM output $y_{\text {tot }}$ (which, as noted in Sec. II, is computed by aggregating the operational level outputs) is written in ANOVA-HDMR form by summing over the operations in Eq. (41), which yields

$$
\begin{aligned}
y_{\text {tot }} & =\sum_{k=1}^{N_{o}} f_{0, k}+\sum_{k=1}^{N_{o}} \sum_{l=1}^{N_{s}} f_{q_{k l}}\left(q_{k l}\right)+\sum_{k=1}^{N_{o}} \sum_{l=1}^{N_{s}} f_{r_{k l}}\left(r_{k l}\right) \\
& +\sum_{k=1}^{N_{o}} \sum_{l=1}^{N_{s}} f_{s_{k l}}\left(s_{k l}\right)+\sum_{k=1}^{N_{o}} \sum_{l=1}^{N_{s}} f_{t_{k l}}\left(t_{k l}\right)+\sum_{k=1}^{N_{o}} \sum_{l=1}^{N_{s}} f_{u_{k l}}\left(u_{k l}\right) \\
& +\sum_{k=1}^{N_{o}} \sum_{l=1}^{N_{s}} f_{v_{k l}}\left(v_{k l}\right)+\text { interaction terms }
\end{aligned}
$$

Now let $q=\left\{q_{k}\right\}_{k=1}^{N_{o}}$ denote the set of fuel-burn inputs across all operations, and let

$$
f_{q}=\sum_{k=1}^{N_{o}} \sum_{l=1}^{N_{s}} f_{q_{k l}}\left(q_{k l}\right)
$$

be the sum of all the single-factor functions of all segment fuel-burn inputs, then Eq. (45) is written as

$$
y_{\mathrm{tot}}=f_{0}+f_{q}+f_{r}+f_{s}+f_{t}+f_{u}+f_{v}+\text { interaction terms }
$$

where $f_{0}=\Sigma_{k=1}^{N_{o}} f_{0, k}$ is the expected value of $y_{\text {tot }}$, and the functions $f_{r}, f_{s}, f_{t}, f_{u}$, and $f_{v}$ are defined analogously to $f_{q}$. Squaring and integrating Eq. (46) gives

$$
\begin{aligned}
\operatorname{var}\left(y_{\mathrm{tot}}\right):= & D=D_{q}+D_{r}+D_{s}+D_{t}+D_{u}+D_{v} \\
& + \text { interaction partial variances }
\end{aligned}
$$

which may also be written as

$$
\begin{aligned}
D & =\sum_{k=1}^{N_{o}} D_{q_{k}}+\sum_{k=1}^{N_{o}} D_{r_{k}}+\sum_{k=1}^{N_{o}} D_{s_{k}}+\sum_{k=1}^{N_{o}} D_{t_{k}}+\sum_{k=1}^{N_{o}} D_{u_{k}}+\sum_{k=1}^{N_{o}} D_{v_{k}} \\
& + \text { interaction partial variances }
\end{aligned}
$$

The total-effect sensitivity index for $q$, denoted $\tau_{q}$, represents the relative contribution to the variance $D$ of all fuel-burn inputs over all operations and segments. As in Eq. (30), we write

$$
\tau_{q}=1-\frac{D_{q^{c}}}{D}
$$

where $D_{q^{c}}$ is the sum of the variances due to all main-effect terms and interaction-effect terms that do not involve fuel burn. By breaking this expression into a sum over operations and using the fact that $D=\Sigma_{k=1}^{N_{o}} D_{y_{k}}$, the expression (49) can be written as

$$
\tau_{q}=1-\frac{\sum_{k=1}^{N_{o}}\left(1-\tau_{q_{k}}\right) D_{y_{k}}}{\sum_{k=1}^{N_{o}} D_{y_{k}}}
$$

where $\tau_{q_{k}}$ is the total-effect sensitivity index for $q_{k}$, the fuel-burn inputs over operation $k$. A similar derivation for main-effect sensitivity indices leads to 

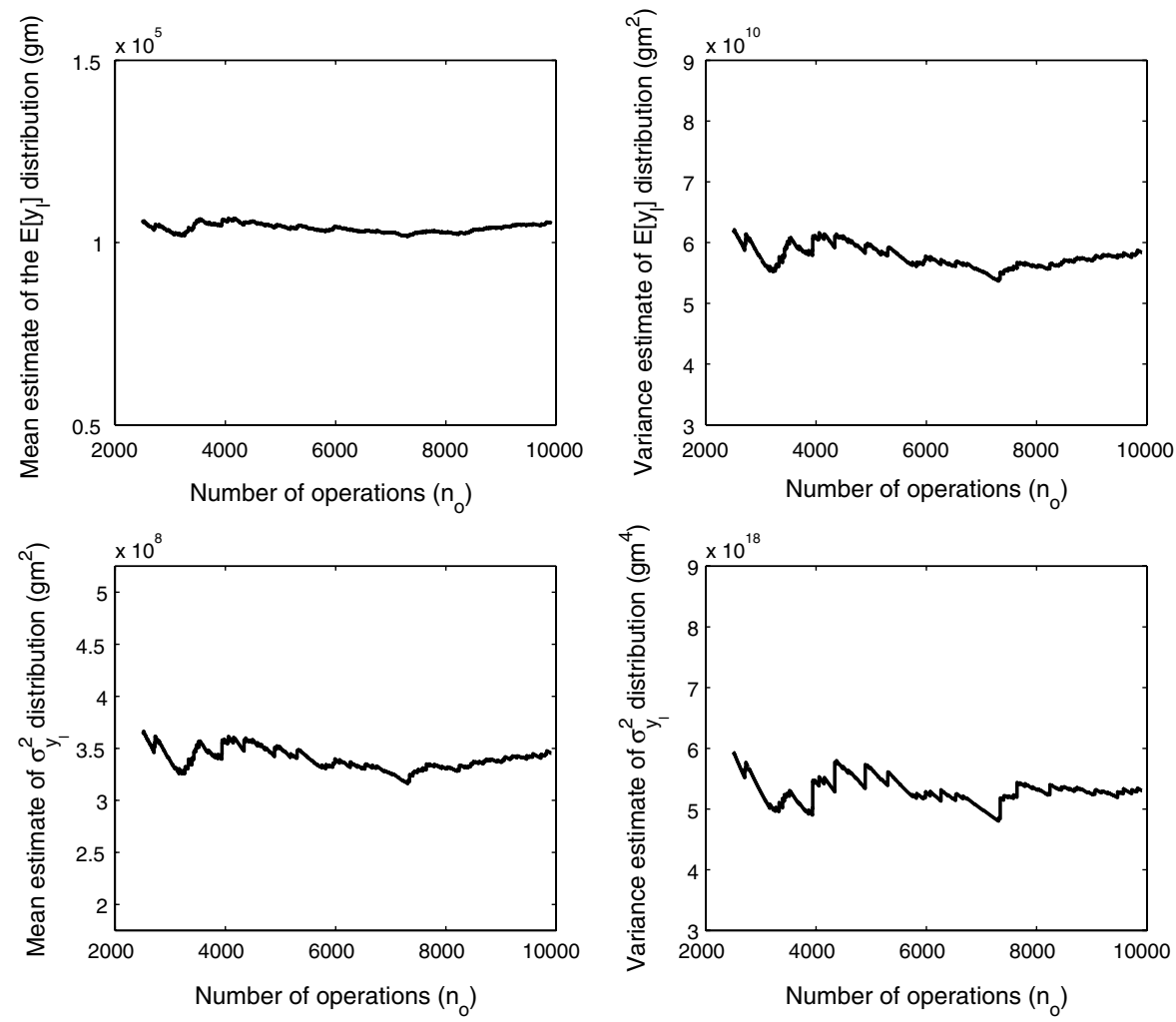

Fig. 4 Estimates of $\mu_{\mathbb{E}}, \sigma_{\mathbb{E}}^{2}, \mu_{\sigma^{2}}$, and $\sigma_{\sigma^{2}}^{2}$ as the number of operations in the surrogate model, $n_{o}$, increases from 2500 to 9914.

$$
S_{q}=\frac{\sum_{k=1}^{N_{o}} S_{q_{k}} D_{y_{k}}}{\sum_{k=1}^{N_{o}} D_{y_{k}}}
$$

As was the case for the expected values of $\mathrm{NO}_{x}$ emissions on the operational level in Sec. III.A, the terms in Eqs. (50) and (51) can be considered as samples from distributions. Therefore, to estimate the sensitivity indices given by Eqs. (50) and (51), we apply the same process used to arrive at Eq. (20) from Eq. (18). In Eq. (1), for example, the distribution of the numerator, $\Sigma_{l=1}^{N_{o}} S_{q_{l}} D_{y_{l}}$, is equal to $N_{o} \mathbb{E}\left[S_{q l} D_{y l}\right]$, which may be estimated from $\left(N_{o} / n_{o}\right) \Sigma_{l \in \mathcal{O}}\left[S_{q l} D_{y l}\right]$. Just as in Eq. (36), this estimate is normally distributed and converges to a single value when $n_{o}=N_{o}$. To estimate confidence intervals for $\tau_{q}$ and $S_{q}$, we sample from distributions of the numerators and denominators to estimate the intervals empirically. It should be noted here that the confidence intervals computed for $\tau_{q}$ and $S_{q}$ will be conservative due to the fact that the numerator and denominator terms in both Eqs. (50) and (51) are positively correlated. By not
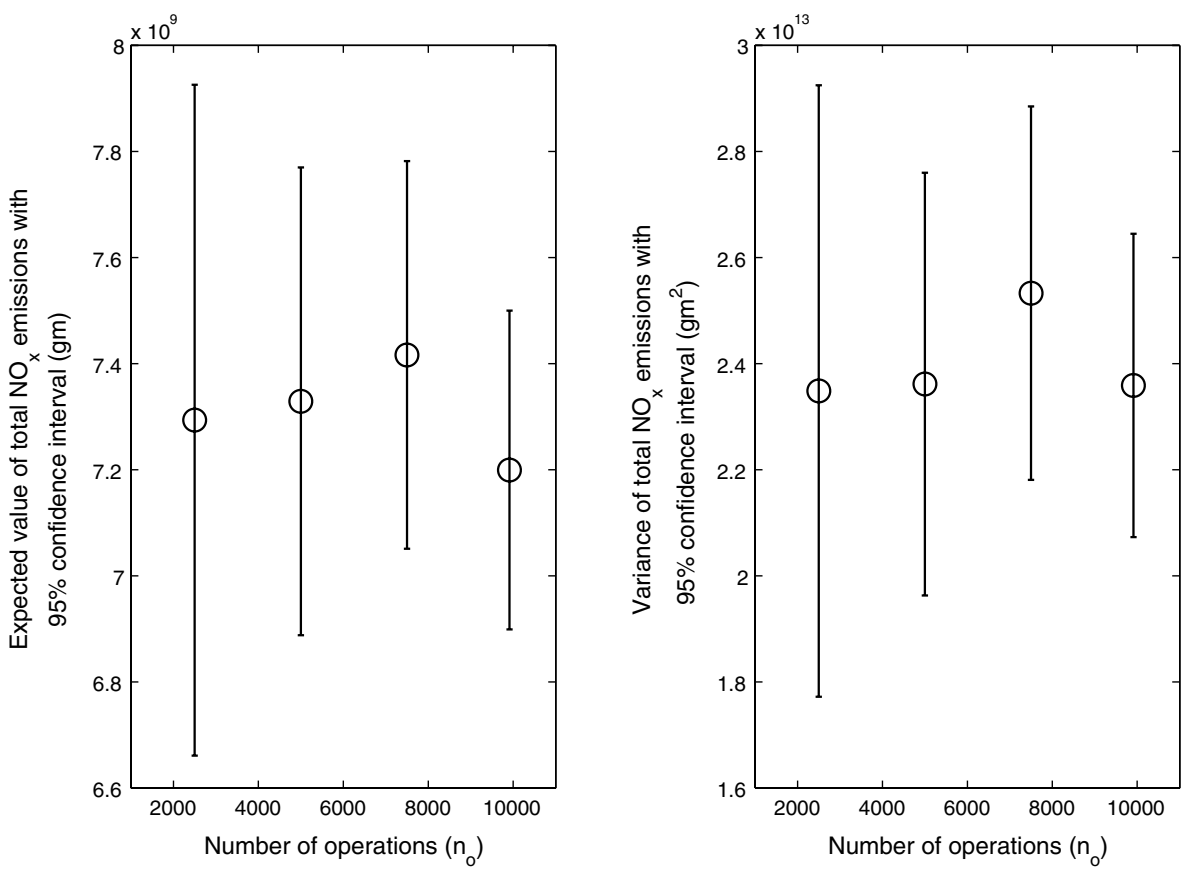

Fig. $595 \%$ confidence intervals of the mean and variance of total $\mathrm{NO}_{x}$ emissions computed with surrogate models of 2500, 5000, 7500, and 9914 operations. 

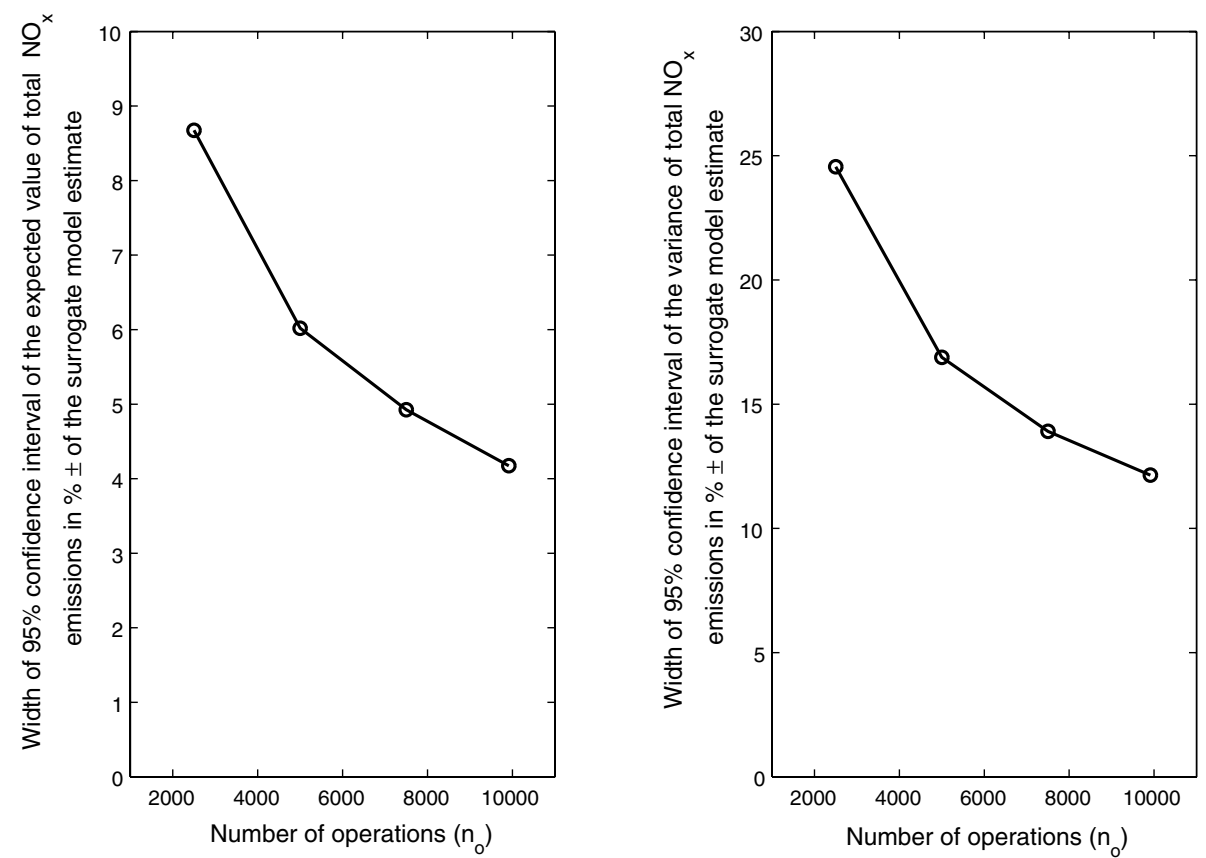

Fig. $695 \%$ confidence interval widths, in terms of percentage \pm of the estimated value, for the mean and variance of total $\mathrm{NO}_{x}$ emissions for a full run of the representative day as $n_{o}$ increases from 2500 to 9914.

including the correlation in the estimation of the confidence intervals, the estimate of the lower endpoint will be less than the true lower endpoint and the estimate of the upper endpoint will be greater than the true upper endpoint. This is due to the fact that the numerator in each equation must be less than or equal to the denominator in each equation, which leads to conservative intervals when the positive correlation term is not included. Results from applying this method to the AEM sensitivity indices for the total emissions of $\mathrm{NO}_{x}$ are presented in the following subsection.

\section{Results}

A typical analysis run of the AEM consists of all operations conducted on a particular day that is considered a reasonable representative of all operations from a particular year. These days are referred to as representative days. The full AEM run for the representative day for the year 2005, which is the AEM model we consider here, has $N_{o}=68,343$ operations. Each of these operations requires a Monte Carlo simulation to calculate operation-level emissions outputs that are then aggregated, as shown in Eq. (33) to produce the overall AEM output, $y_{\text {tot }}$. For the computational resources available for this study, a single model evaluation for one operation takes approximately $2.31 \times 10^{-4} \mathrm{~s}$. To perform both uncertainty and sensitivity analysis for a single operation requires 13 separate Monte Carlo simulations $(2 n+1$ simulations, where $n$, which is the dimension of the input space, is 6 for the AEM), each of which consisted of 10,000 model evaluations in this study. Thus, running each operation of the AEM representative day for 2005 to perform uncertainty and sensitivity analysis on $y_{\text {tot }}$, would take approximately $570 \mathrm{~h}$, which is computationally expensive, especially if many different policy scenarios are to be considered. As will be shown in the following subsections, the methods presented in Sec. III can be used to perform both uncertainty and sensitivity analyses on the AEM representative day with a surrogate model consisting of a randomly chosen subset of operations, while maintaining quantitative rigor in the analyses in a manner that is computationally efficient.

\section{AEM Surrogate Results for Decision-Making Uncertainty Analysis}

To estimate the confidence intervals for the mean and variance of the total $\mathrm{NO}_{x}$ emissions from the representative day, 9914 operations

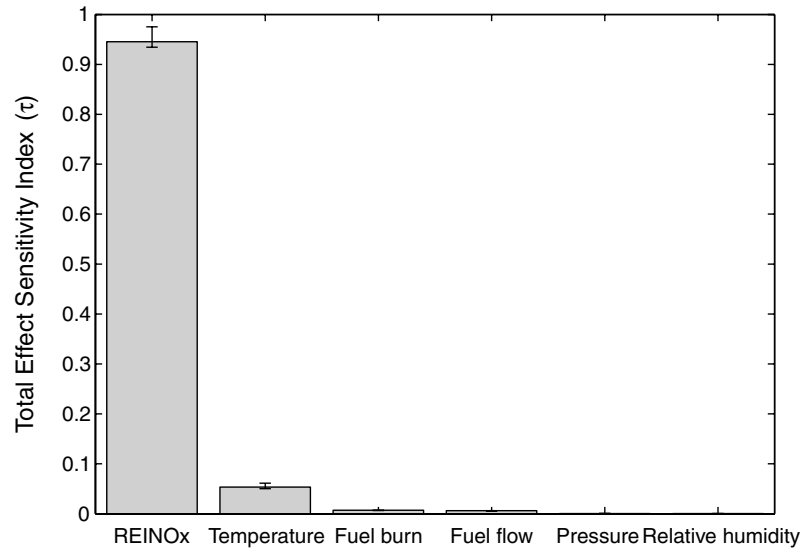

AEM Factors

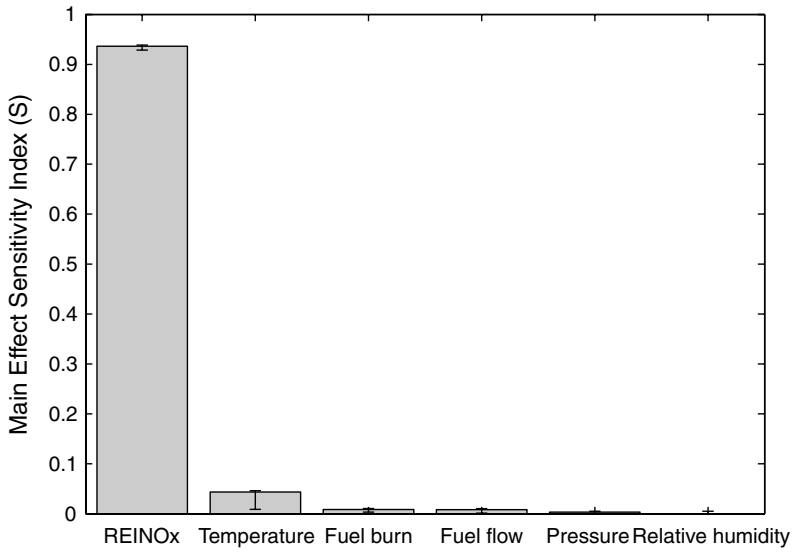

AEM Factors

Fig. 7 Total- and main-effect sensitivity indices for the $\mathrm{AEM} \mathrm{NO}_{x}$ output as evaluated using a surrogate model consisting of 5000 operations. The error bars show the $95 \%$ confidence intervals for each index. 


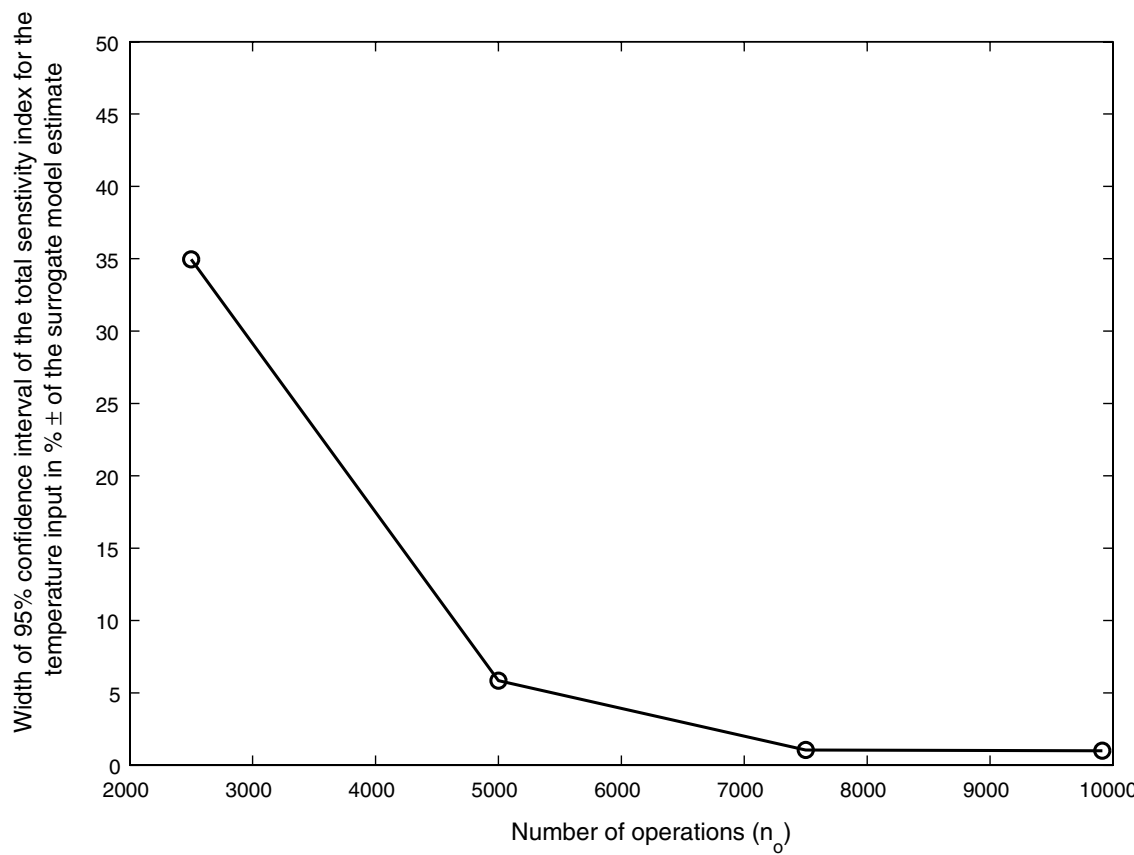

Fig. $895 \%$ confidence interval width, in terms of percentage \pm of the surrogate model sensitivity index estimate, for the total-effect sensitivity index of the temperature input as $n_{o}$ increases from 2500 to 9914.

were chosen randomly, without replacement, from the full set of operations. As noted previously, a 10,000-iteration Monte Carlo simulation was run for each of the sampled operations, the results of which were used to compute operation-level $\mathrm{NO}_{x}$ means and sample variances. These values were then used to estimate the expected value of the distribution of operation-level expected values of $\mathrm{NO}_{x}$ emissions, $\mu_{\mathbb{E}}$; the variance of the expected value of the distribution of operation-level expected values of $\mathrm{NO}_{x}$ emissions, $\sigma_{\mathbb{E}}^{2}$; the expected value of the distribution of operation-level variances of $\mathrm{NO}_{x}$ emissions, $\mu_{\sigma^{2}}$; and the variance of the distribution of operationlevel variances of $\mathrm{NO}_{x}$ emissions, $\sigma_{\sigma^{2}}^{2}$. As was noted in Sec. III, these estimates are necessary for constructing the confidence intervals for the expected value and variance of the total $\mathrm{NO}_{x}$ emissions of the full AEM and uncertainty in these estimates has been neglected. Figure 4 shows the behavior of these estimates as the number of operations in the subset, $n_{o}$, is increased from 2500 to 9914 operations.

The confidence intervals (95\%) for the mean and variance of the total $\mathrm{NO}_{x}$ emissions computed at values of $n_{o}$ of 2500, 5000, 7500, and 9914, are presented in Fig. 5 . Figure 6 presents the dependence of the confidence interval widths, in terms of percentage \pm of the surrogate model estimated values, for the mean and variance of total $\mathrm{NO}_{x}$ emissions for a full run of the representative day as $n_{o}$ increases from 2500 to 9914 . These results show that by applying the surrogate modeling methodology described in Sec. III for uncertainty analysis in support of decision-making, confidence intervals for the mean and variance of total $\mathrm{NO}_{x}$ emissions for the representative day can be constructed. These confidence intervals are quantitatively rigorous

Table 2 95\% confidence intervals of the total- and main-effect sensitivity indices for each input of the AEM for a full run of the representative day computed with a surrogate model of 9914 operations

\begin{tabular}{lcccc}
\hline \hline & \multicolumn{2}{c}{$\begin{array}{c}\text { Total-effect sensitivity } \\
\text { index }\end{array}$} & \multicolumn{2}{c}{$\begin{array}{c}\text { Main-effect sensitivity } \\
\text { index }\end{array}$} \\
\cline { 2 - 5 } Input & Lower & Upper & Lower & Upper \\
\hline REINO $_{x}$ & 0.935 & 0.950 & 0.935 & 0.940 \\
Temperature & 0.050 & 0.053 & 0.008 & 0.060 \\
Fuel burn & 0.006 & 0.007 & 0.003 & 0.010 \\
Fuel flow & 0.005 & 0.006 & 0.001 & 0.010 \\
Pressure & 0.000 & 0.001 & 0.000 & 0.005 \\
Relative humidity & 0.000 & 0.000 & 0.000 & 0.000 \\
\hline \hline
\end{tabular}

and display predictable convergence behavior that can be used to determine optimum tradeoffs between tighter intervals and longer run times.

\section{AEM Surrogate Results for Model Development Sensitivity Analysis}

The total- and main-effect sensitivity indices were computed using the Sobol' method described in Sec. II, applied to a surrogate model of $n_{o}=5000$ operations sampled from the representative day. The resulting total- and main-effect sensitivity indices are shown in Fig. 7. These results reveal that factors such as pressure and relative humidity can potentially be fixed for certain analyses since their total-effect sensitivity indices are low, and that factors such as the reference emissions index of $\mathrm{NO}_{x}$ and temperature should be the focus of any future research aimed at trimming the variability in total $\mathrm{NO}_{x}$ emissions estimates from the AEM, since their main-effect sensitivity indices are highest.

These sensitivity results give valuable insight to guide model development; however, the question arises whether different conclusions might be drawn if the full model were used in place of the surrogate. In this situation, it is computationally impractical to use the full $N_{o}=68,343$ operations; however, the sensitivity results computed with the surrogate of $n_{o}=5000$ operations can be rigorously bounded with confidence intervals using the methodology of Sec. III.B. These confidence intervals were constructed by using a 10,000-iteration Monte Carlo simulation to compute each operationlevel global sensitivity index required in Eqs. (50) and (51). The intervals are shown for each sensitivity index in Fig. 7 .

Figure 8 shows the convergence behavior of the total-effect sensitivity index of the temperature input. The convergence behavior of the other sensitivity indices is similar. Table $\underline{2}$ gives confidence intervals $(95 \%)$ for the total- and main-effect sensitivity indices for each input of the AEM for a full run of the representative day computed with a surrogate model of 9914 operations.

These results show that by applying the surrogate modeling methodology described in Sec. III for model development sensitivity analysis, confidence intervals for the global sensitivity indices of total $\mathrm{NO}_{x}$ emissions for the full representative day can be constructed from a subset of operations. Just as for the confidence intervals constructed to support decision-making uncertainty analysis, these confidence intervals are quantitatively rigorous and display convergence behavior that can be used to determine optimum tradeoffs between tighter intervals and longer run times. 


\section{Limitations and Additional Sources of Error}

The surrogate modeling methodology developed here for the general class of models given by Eq. (16) and demonstrated on the AEM is applicable only if several aforementioned assumptions are met. Those assumptions were independence of model inputs, randomness of model inputs, a large number of random quantities being summed, and satisfaction of the Lyapunov condition by those random quantities. As mentioned in Sec. II.C, the assumptions of independence and randomness in the methods developed here are assumed to be features of the full model, and thus are not restrictions imposed by the surrogate model formulation. If they are not features of the full model, the approach taken here may not be applicable, and analysts may wish to consider the $m$-dependent central limit theorem [21], which for certain situations permits relaxing the independence assumption. For models that fit the form given by Eq. (16) but do not involve summing more than about 30 independent random quantities, we must recognize that the assumption of normality in the resulting sum may not be a good one, and confidence intervals should be estimated with recourse to the Student's $t$-distribution or bootstrapping techniques. The constraint imposed by the Lyapunov condition is easily met in most situations since many examples governed by the model class given by Eq. (16), such as those given in Sec. II.C, consist of random quantities with finite variances whose sum grows without bound as more quantities are added.

Though the surrogate model estimates and their associated confidence intervals presented in Sec. IV.D are considered rigorous, they are only rigorous in the sense of how they approximate the results that would be obtained using the full model. Additional sources of error that exist and would be quantifiable if comparison to reality were possible, include the error associated with number of Monte Carlo model evaluations used to compute quantities such as the expected value of operation-level $\mathrm{NO}_{x}$ emissions, and the fact that the number of random quantities being summed, though large, is not infinite, and thus there is an approximation associated with assuming normality. Since these errors occur for both the surrogate and full models, the errors are not quantified in the surrogate estimates, since the goal of the surrogate modeling methodology was to estimate the outputs of the full model.

\section{Conclusions}

We have presented a novel surrogate modeling methodology designed specifically for supporting decision-making uncertainty analysis and sensitivity analysis for model development for a largescale aviation environmental policy-making model. The surrogate modeling methods developed here allowed for construction of rigorous confidence intervals for metrics that are useful for supporting decision-making (e.g., output means and variances), and for global sensitivity indices, which are useful for informing future research efforts aimed at furthering the development of a model, for a situation where running the analyses on a full model was impractical. Furthermore, the methodology provides predictable convergence behavior of confidence interval widths from the surrogate model estimates, which allows for informed tradeoffs between computation time and uncertainty in the estimation of the various metrics. Here, model structure was exploited to invoke the central limit theorem to derive the confidence intervals; however, the method is more generally applicable using bootstrapping techniques.

\section{Acknowledgments}

This work was supported by the Office of Environment and Energy, U.S. Federal Aviation Administration (FAA) under contract no. DTFAWA-05-D-00012, Task Order 0002, managed by Maryalice Locke. Any opinions, findings, and conclusions or recommendations expressed in this material are those of the author(s) and do not necessarily reflect the views of the FAA. The authors acknowledge the support of R. Cointin, FAA Assessment Program Lead.

\section{References}

[1] Cacuci, D., Sensitivity and Uncertainty Analysis: Volume I, Theory, Chapman \& Hall/CRC, Boca Raton, FL, 2003.

[2] Chan, K., Saltelli, A., and Tarantola, S., "Sensitivity Analysis of Model Output: Variance-Based Methods Make the Difference," Proceedings of the 1997 Winter Simulation Conference, IEEE Computer Society, Washington, D.C., 1997, pp. 261-268.

[3] Homma, T., and Saltelli, A., "Importance Measures in Global Sensitivity Analysis of Nonlinear Models," Reliability Engineering and System Safety, Vol. 52, No. 1, 1996, pp. 1-17.

[4] Saltelli, A., Ratto, M., Andres, T., Campolongo, F., Cariboni, J., Gatelli, D., Saisana, M., and Tarantola, S., Global Sensitivity Analysis: The Primer, Wiley, West Sussex, England, U.K., 2008.

[5] Grimmet, G., and Stirzaker, D., Probability and Random Processes, 3rd ed., Oxford Univ. Press, Oxford, 2006.

[6] Sobol', I., "Theorems and Examples on High Dimensional Model Representation," Reliability Engineering and System Safety, Vol. 79, No. 2, 2003, pp. 187-193.

[7] Saltelli, A., and Bolado, R., "An Alternative Way to Compute Fourier Amplitude Sensitivity Test (FAST)," Computational Statistics and Data Analysis, Vol. 26, No. 4, 1998, pp. 445-460. doi:10.1016/S0167-9473(97)00043-1

[8] Morris, M., "Factorial Sampling Plans for Preliminary Computational Experiments," Technometrics, Vol. 33, No. 2, 1991, pp. 161-174. doi: $10.2307 / 1269043$

[9] Saltelli, A., Tarantola, S., and Chan, K., "A Quantitative ModelIndependent Method for Global Sensitivity Analysis of Model Output," Technometrics, Vol. 41, No. 1, 1999, pp. 39-56. doi: $10.2307 / 1270993$

[10] Eldred, M., Giunta, S., and Collis, S., "Second-Order Corrections for Surrogate-Based Optimization with Model Hierarchies," 10th AIAA/ ISSMO Multidisciplinary Analysis and Optimization Conference, AIAA Paper 2004-4457, 2004.

[11] Venter, G., Haftka, R., and Starnes, J., "Construction of Response Surface Approximations for Design Optimization," AIAA Journal, Vol. 36, No. 12, 1998, pp. 2242-2249. doi: $10.2514 / 2.333$

[12] Antoulas, A. C., and Sorensen, D., Approximation of Large-Scale Dynamical Systems, Society for Industrial and Applied Mathematics, Philadelphia, 2005.

[13] Alexandrov, N., Lewis, R., Gumbert, C., Green, L., and Newman, P., "Approximation and Model Management in Aerodynamic Optimization with Variable-Fidelity Models," Journal of Aircraft, Vol. 38, No. 6, 2001, pp. 1093-1101. doi: $10.2514 / 2.2877$

[14] Alexandrov, N., Nielsen, E., Lewis, R., and Anderson, W., "First-Order Model Management in Aerodynamic Optimization with VariableFidelity Models," 8th AIAA/USAF/NASA/ISSMO Symposium on Multidisciplinary Analysis and Optimization, AIAA Paper 2000-4886, 2000.

[15] Choi, S., Grandhi, R., Canfield, R., and Pettit, C., "Polynomial Chaos Expansion with Latin Hypercube Sampling for Estimating Response Variability," AIAA Journal, Vol. 42, No. 6, 2004, pp. 1191-1198. doi: $10.2514 / 1.2220$

[16] Robinson, T., Eldred, M., Willcox, K., and Haimes, R., "Strategies for Multi-Fidelity Optimization with Variable Dimensional Hierarchical Models," 2nd MDO Specialists Conference, AIAA Paper 2006-1819, Newport, RI, 2006.

[17] Simpson, T., Peplinski, J., Koch, P., and Allen, J., "Metamodels for Computer-Based Engineering Design: Survey and Recommendations," Engineering with Computers, Vol. 17, No. 2, 2001, pp. 129-150. doi:10.1007/PL00007198

[18] Messac, A., and Mullur, A., "A Computationally Efficient Metamodeling Approach for Expensive Multiobjective Optimization," Optimization and Engineering, Vol. 9, No. 1, 2008, pp. 37-67. doi:10.1007/s11081-007-9008-0

[19] Karr, A., Probability, Springer, New York, 1993.

[20] Lipschutz, S., Schiller, J., and Srinivasan, R., Schaum's Outline of Theory and Problems of Beginning Finite Mathematics, McGraw-Hill Professional, New York, 2004.

[21] Berk, K., "A Central Limit Theorem for $m$-Dependent Random Variables with Unbounded $m$," The Annals of Probability, Vol. 1, No. 2, 1973, pp. 352-354. doi:10.1214/aop/1176996992

[22] Horvitz, D., and Thompson, D., "A Generalization of Sampling Without Replacement from a Finite Universe," Journal of the American Statistical Association, Vol. 47, No. 260, 1952, pp. 663-685. doi: $10.2307 / 2280784$ 
[23] Kutner, M., Nachtsheim, C., Neter, J., and Li, W., Applied Linear Statistical Models, 5th ed., McGraw-Hill, Boston, 2005.

[24] Baughcomb, S., Henderson, S., and Tritz, T., "Scheduled Civil Aircraft Emissions Inventories for 1976 and 1984: Database Development and Analysis," NASA CR-4722, 1996.

[25] Kim, B., Fleming, G., Lee, J., Waitz, I., Clarke, J., Balasubramanian, S., et al., "System for Assessing Aviation's Global Emissions (SAGE),
Part 1: Model Description and Inventory Results," Transportation Research, Part D (Transport and Environment), Vol. 12, No. 5, 2007, pp. 325-346.

doi:10.1016/j.trd.2007.03.007

A. Messac Associate Editor 